\title{
Performance Analysis of an Optical Packet Switch Employing Full/Limited Range Share Per Node Wavelength Conversion
}

\author{
Nail Akar and Ezhan Karasan \\ Electrical and Electronics Eng. \\ Bilkent University, Bilkent 06800, Ankara, Turkey
}

\author{
Giovanni Muretto and Carla Raffaelli \\ DEIS - University of Bologna \\ Bologna, Italy
}

\begin{abstract}
In this paper, we study an asynchronous optical packet switching node equipped with a number of limited range or full range wavelength converters shared per node. The packet traffic is realistically modeled by a superposition of a finite number of on-off sources as opposed to the traditional Poisson model which ignores the limited number of ports on a switch. We both study circular and non-circular limited range wavelength conversion schemes. In our simulations, we employ the far conversion policy where the optical packet is switched onto the farthest available wavelength in the tuning range, which is known to outperform the random conversion policy. We propose an approximate analytical method based on block tridiagonal Markov chains and fixed point iterations to solve for the blocking probabilities in share per node wavelength conversion systems. The method provides an accurate approximation for full range systems and acceptable results for limited range systems.
\end{abstract}

\section{INTRODUCTION}

Two packet-based optical switching paradigms have recently been introduced to make more efficient use of bandwidth (as opposed to circuit-based networks): Optical Packet Switching (OPS) [1] and Optical Burst Switching (OBS) [2]. In this paper, we study the performance of an optical packet/burst switch employing full/limited range share per node wavelength conversion. As far as this work is concerned, we do not differentiate between OPS and OBS and for the sake of simplicity, we will use the common term "(optical) packet" and "(optical) packet switching" to refer to a packet/burst and the data planes of OPS/OBS, respectively.

In synchronous (i.e., time-slotted) optical packet switching networks, packet lengths are fixed and therefore there is a need for costly synchronization equipment. In asynchronous (i.e., unslotted) networks, optical packet lengths are variable and packet arrivals need not be aligned. Moreover, asynchronous packet switching is a more natural fit for supporting client networks carrying variable sized data packets. e.g., IP networks. In this paper, we focus on asynchronous optical packet switching.

In OPS networks, contention arises when there are two or more incoming packets contending for the same output wavelength. The first choice for contention resolution is to use Tunable Wavelength Converters (TWC), although other resolution mechanisms also exist, for example Fiber Delay Lines (FDL) and deflection routing [2]. In Full Wavelength
Conversion (FWC), we have a TWC for each wavelength channel. In Partial Wavelength Conversion (PWC), we have TWC sharing amongst a number of wavelength channels. Depending on how TWC sharing takes place, a number of architectures have been proposed for PWC. On one end, we have dedicated TWC banks for each output fiber line, called the Share Per Line (SPL) architecture [3]. On the other end, TWCs may be collected as a single converter pool for more efficient converter sharing across all fiber lines, which is referred to as the Share Per Node (SPN) architecture [3]. However, there are different architectures for TWCs which can be classified with respect to their tuning ranges. Full Range TWCs (FR-TWC) do not have tuning range limitations and they can convert an incoming wavelength to any other wavelength available in the system. In limited range wavelength conversion, a packet arriving on a wavelength can be converted to a fixed set of wavelengths above and below the original wavelength. Such converters are called Limited Range TWCs (LR-TWC) [4]. For LRTWCs, conversion degree $d$ is defined as the total number of wavelengths available on both sides of the original wavelength for conversion purposes. LR-TWCs are also classified on the basis of the neighboring relationship for the wavelengths at the boundaries. In circular conversion, we assume the wavelengths are wrapped around to form a circle so that the wavelengths at the boundaries become neighbors. On the other hand, in noncircular-type limited range conversion, we do not allow wraparound and the conversion ranges for wavelengths close to the boundaries are reduced in size. The difference between circular and non-circular wavelength conversion is presented in Fig. 1, that illustrates the adjacency set of each input wavelength in case of 8 wavelength channels and $d=2$. In this paper, we both study circular and non-circular conversion schemes.

The focus of the current paper is on the performance analysis of a bufferless asynchronous optical packet switch employing SPN LR-TWCs (see Fig. 2 for two such architectures). In this scenario, the packet switching node is equipped with $N$ input/output fiber interfaces each carrying $M$ wavelengths. We also have $R$ LR-TWCs grouped together in a single bank so that an incoming packet can exploit any of the TWCs irrespective of the destination fiber line. The optical packet traffic is modeled as follows. For each input wavelength channel (there are overall $K=M N$ input channels), there is 


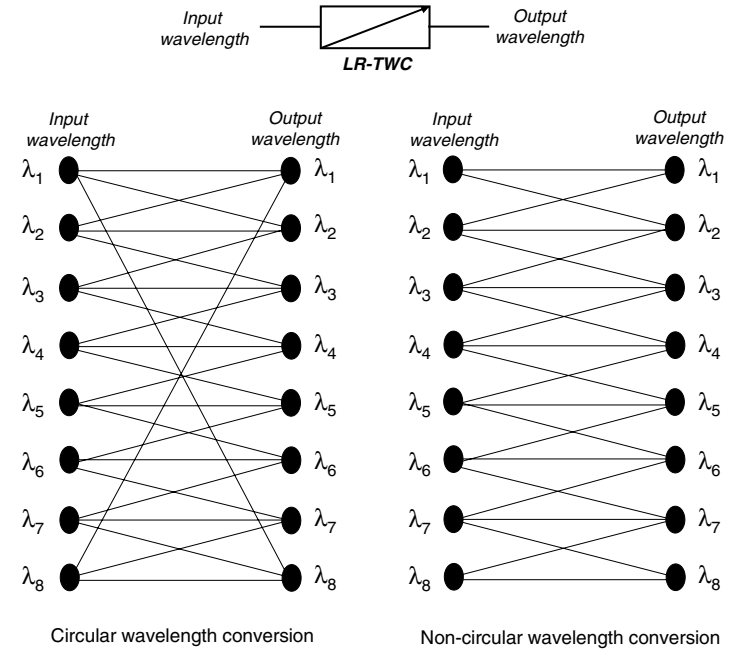

Fig. 1. Circular and non-circular conversion scheme depicted for $d=2$ for a WDM system with 8 channels
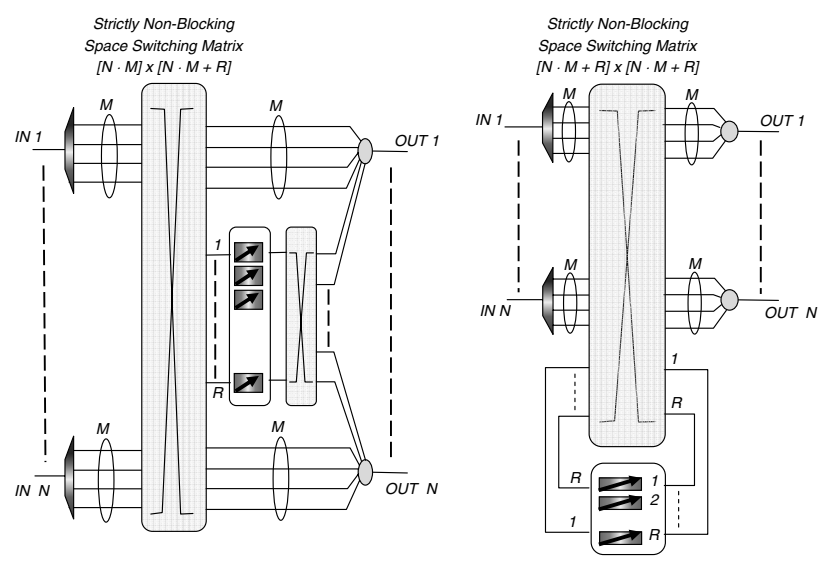

Fig. 2. Two switching node architectures with $N$ input and output fibers, $M$ wavelengths per fiber and limited number $R$ of LR-TWCs shared per node.

an on-off source governing the input packet traffic. In this model, either the input channel is on (an optical packet is being transmitted on that channel) or the input channel is off (the input channel is idle). We assume in this study that the on and off times for each source are exponentially distributed with common means $1 / \mu$ and $1 / \lambda$, respectively. The offered load to the system is $\rho=\frac{\lambda}{(\lambda+\mu)}$. We also assume that each optical packet will be destined to one of the output fiber lines with probability $1 / N$. Therefore, the offered load for each output fiber line is $\rho$, i.e., symmetric loading. The generalization to more general traffic scenarios where loading on different output fiber lines is different, i.e., asymmetric loading, is left for future research. We call this traffic model a finite population traffic model since at any time there will at most be $K=N M$ packets destined to a particular output fiber line. This model is also known as the Engset model in the teletraffic literature and has been used for traffic modeling in optical packet switched networks [5]. The Engset model is different from infinite population models, e.g., Poisson model, where there may not be any upper limit on the maximum number of packets destined to a fiber line at a given time. In this respect, finite population models provide a better fit for switching systems with limited number of interfaces.

For SPL type converter sharing in asynchronous switching systems, the first exact algorithm is proposed in [6] that relies on the steady-state solution of a Markov chain and exploiting the block tridiagonal structure of the underlying infinitesimal generator. Recently, a similar CTMC-based analysis is proposed in [7] for the same system and an approximate analytical method is proposed for the SPN converter sharing case using fixed point iterations. Both studies above assume full range but shared wavelength conversion. Limited range conversion studies are rather rare. In [8], the authors provide an approximate method for SPL type converter sharing using LRTWCs again using Markov chains and show that far conversion policies provide better performance when compared with random or near conversion policies for SPL type conversion. In [9], a product form solution is given for the special cases of $d=2$ and $d=4$ whereas an approximation technique is presented for more general scenarios for SPL type converter sharing. Studies on limited range wavelength conversion but for synchronous optical packet switching systems are more mature [10]. Recently, a Markovian analysis is carried out in [11] for synchronous switching systems employing SPN type LR-TWCs. The contribution of the current paper is two fold. First, we use the idea of fixed point iterations of [7] but for the more realistic on-off traffic model (as opposed to Poisson models) for studying SPN type converter sharing using full range TWCs. While doing so, we benefit from the block tridiagonal structure of the generators that arise using a technique similar to one introduced in [6]. Secondly, we use a similar approximation as in [8] to deal with limited range conversion. Combining these two methods provides us with a mechanism to analyze switching systems employing full/limited range share per node wavelength conversion.

The outline of the paper is as follows. The approximate analytical method is presented in Section II to calculate packet blocking probabilities in the switching system of interest. Numerical results are presented in Section III. We conclude in the final section.

\section{Analytical Method}

Let us first concentrate on a single output fiber (tagged fiber) which consists of $M$ wavelength channels. Recall that the other fibers are statistically equivalent and the stochastic analysis of the tagged fiber will be sufficient for analyzing the entire system. In this case, an incoming optical packet destined to the tagged fiber (with probability $1 / N$ ) is forwarded without conversion if its incoming wavelength is idle on the outgoing link. If the incoming wavelength is occupied then there are two possibilities: if there is an idle wavelength in the tuning range then the packet will be directed to the converter pool or otherwise the packet will be blocked. In the former case, if all the converters are in use then the packet will again be 
blocked otherwise the packet will be directed to the destination fiber using one of the free converters and one of the available wavelengths in the tuning range. For the far conversion policy, the farthest idle wavelength in the tuning range is selected as the outgoing wavelength.

For mathematical analysis, let $i(t)$ and $j(t)$ denote the number of wavelength channels that are in use on the tagged fiber and the number of input wavelength channels that are in the on state, respectively, at time $t$. We assume that the tagged fiber process and the converter process are independent and the tagged fiber process is impacted only through the converter loss probability $p_{b c}$ which is defined as the probability of blocking due to conversion for a packet directed to the converter pool. Under this assumption, the process $\{(i(t), j(t)): t \geq 0\}$ is a Markov process on the state space $S=\{(i, j): 0 \leq j \leq K, 0 \leq i \leq \min (M, j)\}$. To show this, let us assume that the process is in some state $(i, j)$ at time $t$. If a new packet arrives in the interval $(t, t+\Delta t)$ which occurs with probability $(K-j) \lambda \Delta t+O(\Delta t)$ (i.e., $\lim _{\Delta t \rightarrow 0} O(\Delta t) / \Delta t=0$ ) [12], then the packet will be destined to the tagged fiber with probability $1 / N$. Otherwise, the packet is destined to another fiber and the Markov chain governing the tagged fiber will jump to state $(i, j+1)$. When the arriving packet is destined to the tagged fiber, it will require conversion with probability $i / M$; otherwise the packet will be directed to the tagged fiber and the Markov chain will jump to state $(i+1, j+1)$ (or will be blocked when $i(t)=M$ and the visited state will be $(i, j+1))$. When the packet requires conversion, we check the fullness probability of the tuning range denoted by $p_{l r}(i, d)$ as a function of $i$ and $d$ for an incoming packet finding $i$ channels occupied and requiring conversion. However, it is very hard to derive this quantity for which we propose an approximation based on [8]. In this approximation, the conversion range is not the actual $d / 2$ neighborhood of the incoming wavelength in the circular case but is instead taken as a set of arbitrarily selected $d$ wavelengths at each time conversion is to take place. This simpler model captures the impact of degree of conversion but does not accommodate the clustering effect mentioned in [8]. Based on this simpler model, for the circular case we write

$$
p_{l r}^{c}(i, d)= \begin{cases}\frac{i-1}{K-1} \frac{i-2}{K-2} \cdots \frac{i-d}{K-d} & \text { if } i \geq d+1, \\ 0 & \text { if } i \leq d .\end{cases}
$$

Similarly, for the non-circular case and for even $M$

$$
p_{l r}^{n c}(i, d)=2 / N \sum_{k=1}^{M / 2} p_{l r}^{c}(i, \min (d, d / 2+k-1)),
$$

since the two wavelengths at the boundaries can only be converted to $d / 2$ wavelengths, their neighboring wavelengths towards the middle can be converted to $d / 2+1$ wavelengths and so on. The odd $M$ case can also be treated similarly. For full-range wavelength conversion (indicated by the superscript $f r$ ), we do not have a range limit and therefore we have the exact identity

$$
p_{l r}^{f r}(i)=0, \forall i
$$

If the tuning range is not full then the packet is directed to the converter pool comprised of $R$ converters and the packet will either be blocked due to the lack of converters with probability $p_{b c}$ and the visited state will be $(i+1, j)$ or the packet will use one of the free converters so as to be directed to the tagged fiber and the Markov chain will jump to state $(i+1, j+1)$. If a packet departure occurs in the interval $(t, t+\Delta t)$ which occurs with probability $j \mu \Delta t+O(\Delta t)$, then the Markov chain will jump to state $(i-1, j-1)$ with probability $1 / N$ and to $(i-1, j)$ otherwise. It is thus clear that the process $X(t)$ is a Continuous Time Markov Chain (CTMC) and the infinitesimal generator of the CTMC possesses a block-tridiagonal form if the states are properly enumerated as

$$
\begin{aligned}
S= & \{\underbrace{(0,0)}_{\text {level } 0}, \underbrace{(0,1),(1,1)}_{\text {level } 1}, \\
& \underbrace{(0,2),(1,2),(2,2)}_{\text {level } 2}, \cdots, \underbrace{(0, K), \cdots,(M, K)}_{\text {level } K}\} .
\end{aligned}
$$

A numerically stable and efficient solution procedure, the socalled block tridiagonal LU factorization algorithm can then be used to find the stationary solution of the underlying CTMC while taking advantage of the block-tridiagonal structure of the generator [13, pages 174-175]. The dependency (to $K$ ) of the complexity of the block tridiagonal LU factorization algorithm is $O(K)$. This is in contrast with the $O\left(K^{3}\right)$ computational complexity governing the brute force approach. Therefore switching systems with large number of interfaces are not beyond reach as will be shown throughout the numerical examples. We derive two quantities using this model; one of them is $p_{c}$ which denotes the probability than an incoming packet directed to the tagged fiber is also directed to the converter pool and the other one is $p_{b}$, the overall blocking probability. For this derivation, let $x$ be the steady-state vector for the circular case and $x(i, j)$ be the steady-state probability of finding the Markov process at state $(i, j)$ at an arbitrary epoch. We first write

$$
p_{c}=\frac{1}{\Delta} \sum_{j=1}^{K} \sum_{i=1}^{M-1} x(i, j)(K-j)(i / M)\left(1-p_{l r}^{c}(i, d)\right),
$$

where $\Delta=\frac{K \mu}{\lambda+\mu}$. We then write $p_{b}=$

$$
\frac{1}{\Delta}\left(\begin{array}{l}
\sum_{j=1}^{K} \sum_{i=1}^{M-1} x(i, j)(K-j)(i / M)\left(1-p_{l r}^{c}(i, d)\right) p_{b c} \\
+\sum_{j=1}^{K} \sum_{i=1}^{M-1} x(i, j)(K-j)(i / M) p_{l r}^{c}(i, d) \\
+\sum_{j=1}^{K}(K-j) x(M, j)
\end{array}\right) .
$$

Note that the non-circular (full range) case can be solved by replacing the probabilities $p_{l r}^{c}$ by $p_{l r}^{n c}\left(p_{l r}^{f r}\right)$ throughout the entire procedure.

At this point, we can calculate the probabilities $p_{c}$ and $p_{b}$ upon a-priori information about $p_{b c}$. However, the latter quantity also needs to be calculated. We will now show that $p_{b c}$ can be calculated using $p_{c}$ which will lead us to a fixed point iteration. To see this, first note that $p_{c}$ is also the probability that an arriving packet is directed to the converter pool due 
to symmetry amongst fibers. Let us now concentrate on the following problem which consists of $K$ on-off sources with each packet (corresponding to an on time) directed to the pool of $R$ converters with probability $p_{c}$. The blocking probability in this new system gives us $p_{b c}$. For this system, let $i(t)$ and $j(t)$ denote the number of TWCs that are in use and the number of sources that are in the on state, respectively. The process $\{(i(t), j(t)): t \geq 0\}$ is then a Markov process on the state space $S=\{(i, j): 0 \leq j \leq K, 0 \leq i \leq \min (R, j)\}$. To show this, let us assume that the process is in some state $(i, j)$ at time $t$. If a new packet arrives which occurs with rate $(K-j) \lambda$ then the packet will be directed to the converter pool with probability $p_{c}$. The packet will be admitted into the system if $i<R$ and the Markov chain will jump to state $(i+1, j+1)$ or will be blocked when $i(t)=R$ and the visited state will be $(i, j+1)$. If a packet departure occurs (with rate $j \mu)$ then the Markov chain will jump to state $(i-1, j-1)$ with probability $\frac{i}{j}$ or to $(i, j-1)$ with probability $1-\frac{i}{j}$. This shows that the underlying system is Markov and this system again has a block tridiagonal generator. Solving for the steadystate probabilities of finding the system in state $(i, j)$ denoted by $y(i, j)$ using the above-mentioned block tridiagonal $\mathrm{LU}$ factorization algorithm, we finally have

$$
p_{b c}=\frac{1}{\Delta}\left(\sum_{j=R}^{K} y(R, j)(K-j)\right) .
$$

The whole procedure can be summarized as follows.

1. First start with a initial converter blocking probability, say $p_{b c}=0$.

2. Given $p_{b c}$, construct and solve the Markov chain governing the tagged fiber and find $p_{c}$ through Eqn. 4.

3. Given $p_{c}$, construct and solve the Markov chain governing the converter process and find $p_{b c}$ through Eqn. 6. Go back to Step 2 unless the two successive values of $p_{b c}$ are close.

4. Write $p_{b}$ through Eqn. 5 which gives us an approximation for the blocking probability.

\section{NUMERICAL EXAMPLES}

In this numerical example, we first study the accuracy of the proposed analytical method for the full range wavelength conversion case. For this purpose, we first introduce a wavelength conversion percentage ratio parameter $r=100 \frac{R}{N M}$. We then plug the identity (3) into the analytical procedure for fixed $M=8$ and for varying $N$, for two different values of $\rho=0.3,0.7$, and for three different values of $r=6.25 \%, 25 \%$, and $50 \%$. The results are given in Figures 3 and 4.

We first observe that the analytical approach produces very accurate results especially with increasing $N$ since the independence assumption between the fiber process and the converter process is most justified when $N$ is relatively large. Secondly, we observe that there are two effects counteracting each other when we vary $N$; the first one is when we have full conversion, i.e., $r \rightarrow 100 \%$, the blocking probability increases with increasing $N$ since with more interfaces the output

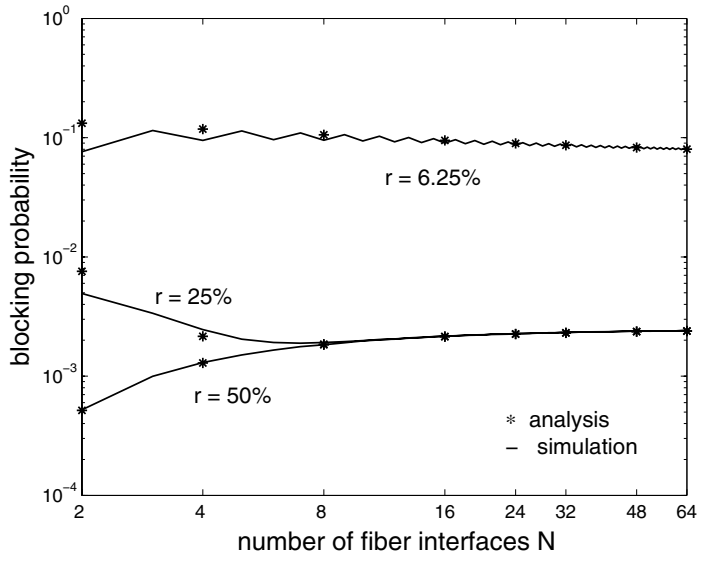

Fig. 3. Blocking probability as a function of the number of interfaces $N$ for an 8-wavelength system with $\rho=0.3$ and for three values of $r=6.25 \%, 25 \%$, and $50 \%$.

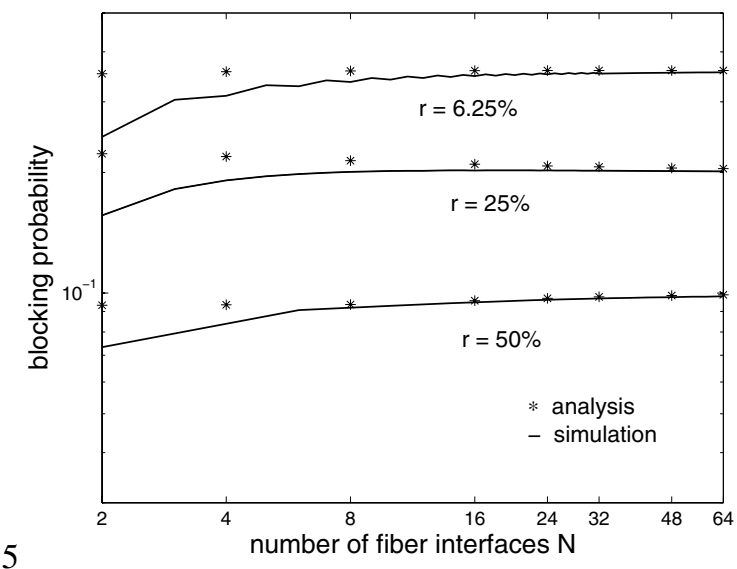

Fig. 4. Blocking probability as a function of the number of interfaces $N$ for an 8-wavelength system with $\rho=0.7$ and for three values of $r=6.25 \%, 25 \%$, and $50 \%$.

contention probability increases as explained in [14]. However, when we have partial wavelength conversion, we have better sharing of converter resources when $N$ increases due to economy of scale which leads to reduced blocking probabilities. These counteracting effects are illustrated in Fig. 3 where the blocking probability decreases (increases) for low (high) conversion ratios and we observe both effects for a moderate conversion ratio when we have low utilization. In Fig. 4, which is for a high utilization, we observe only the former effect and the blocking rate strictly increases with increasing $N$ for different conversion ratios. In the second numerical example, we study the accuracy of the proposed analytical method for limited range wavelength conversion. We plug the identities (1) and (2) for the circular and noncircular cases, respectively, into the analytical procedure for fixed $M=8$ and $\rho=0.3$, but for varying $r$. The results are depicted in Figures 5 and 6 . The analytical procedure underestimates the blocking probabilities but generally gives acceptable results especially for larger $d$. The far conversion policy outperforms the random 


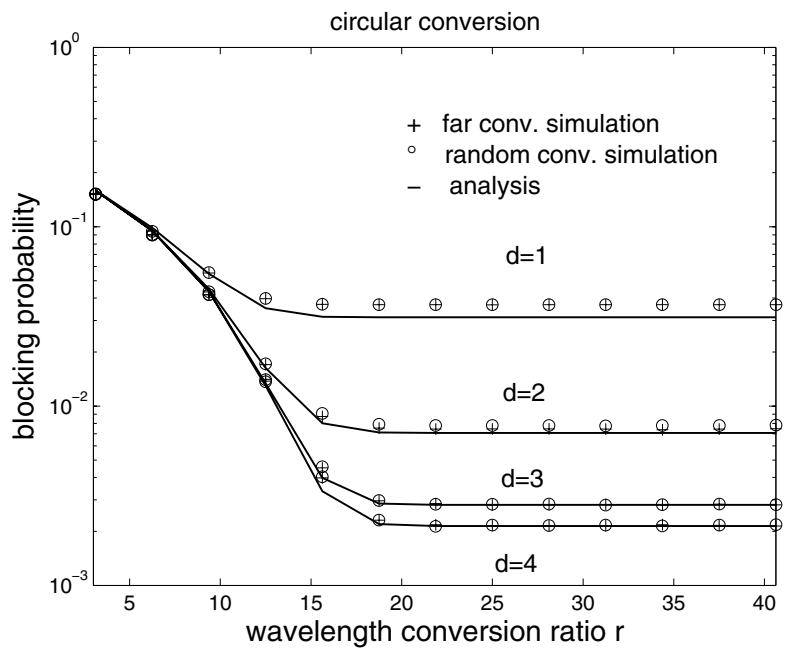

Fig. 5. Blocking probability as a function of the wavelength conversion ratio $r$ for an 8-wavelength circular conversion system with $\rho=0.3$ and for different values of the degree parameter $d$.

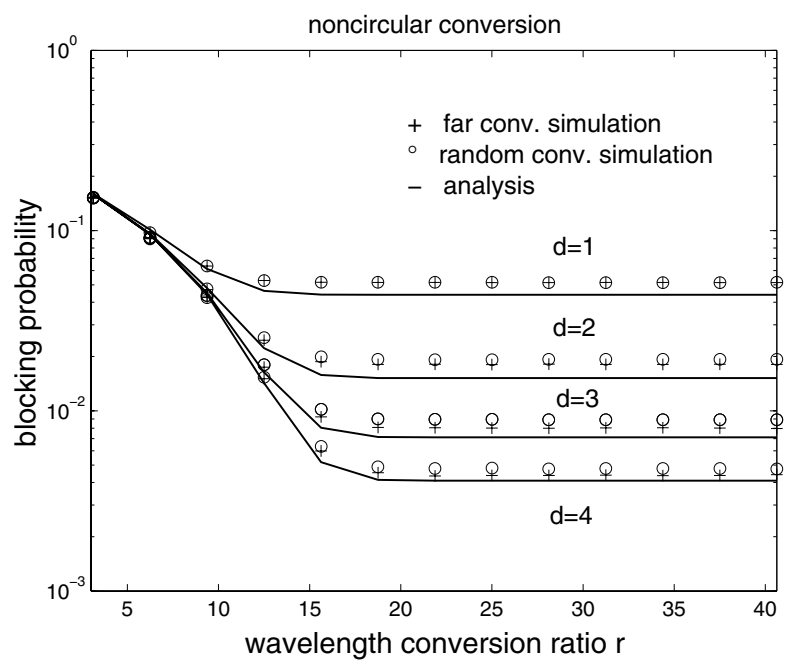

Fig. 6. Blocking probability as a function of the wavelength conversion ratio $r$ for an 8-wavelength noncircular conversion system with $\rho=0.3$ and for different values of the degree parameter $d$.

conversion policy for SPN sharing and the analytical method provides a better approximation to far conversion than random conversion. Irrespective of the values of the degree parameter $d$, the blocking probabilities saturate at around $r=20 \%$ leading us to believe that the use of full wavelength conversion for SPN systems may not be as necessary especially for low loads.

\section{CONCLUSION}

In this paper, we study an asynchronous optical packet switching node equipped with a number of wavelength converters shared per node. We study the full range, limited range circular, and limited range noncircular cases using Markov chains and fixed point iterations. In our approach, we also take into account the finite number of fiber interfaces using the
Engset traffic model. The proposed analytical method provides almost perfect accuracy for full range systems especially for systems with relatively large number of fiber interfaces and acceptable approximations for limited range systems for both circular and noncircular scenarios. With this analytical procedure at hand, we plan on studying different scenarios and providing converter provisioning guidelines.

\section{ACKNOWLEDGMENT}

This work is supported in part by The Science and Research Council of Turkey (Tübitak) under grant no. EEEAG-106E046 and by the European Commission through the Network of Excellence e-Photon/ONe+.

\section{REFERENCES}

[1] P. Gambini, M. Renaud, C. Guillemot, F. Callegati, I. Andonovic, B. Bostica, D. Chiaroni, G. Corazza, S. L. Danielsen, P. Gravey, P. B. Hansen, M. Henry, C. Janz, A. Kloch, R. Krahenbuhl, C. Raffaelli, M. Schilling, A. Talneau, and L. Zucchelli, "Transparent optical packet switching: network architecture and demonstrators in the KEOPS project", IEEE J. Select. Areas Commun., vol. 16, pp. 1245-1259, 1998.

[2] C. Qiao and M. Yoo, "Optical burst switching (OBS) - a new paradigm for an optical Internet”, Jour. High Speed Networks (JHSN), vol. 8, no. 1, pp. 69-84, 1999.

[3] V. Eramo, M. Listanti, and P. Pacifici, "A comparison study on the wavelength converters number needed in synchronous and asynchronous all-optical switching architectures", J. Lightw. Technol., vol. 21, no. 2, pp. 340-355, 2003.

[4] V. Puttasubbappa and H. Perros, "An approximate queueing model for limited-range wavelength conversion in an OBS switch", in Networking, Ontario, Canada, 2005.

[5] H. Overby, "Performance modelling of optical packet switched networks with the engset traffic model", Optics Express, vol. 13, no. 5, pp. 1685$1695,2005$.

[6] N. Akar and E. Karasan, "Exact calculation of blocking probabilities for bufferless optical burst switched links with partial wavelength conversion", in 1st Conference on Broadband Networks (BROADNETS'04), Optical Networking Symposium, 2004, pp. 110-117.

[7] Y. Mingwu, L. Zengji, and W. Aijun, "Accurate and approximate evaluations of asynchronous tunable-wavelength-converter sharing schemes in optical burst-switched networks", J. Lightw. Technol., vol. 23, no. 10, pp. 2807-2815, 2005.

[8] K. Dogan, Y. Gunalay, and N. Akar, "Comparative study of limited range wavelength conversion policies for asynchronous optical packet switching", Journal of Optical Networking, vol. 6, pp. 134-145, 2007.

[9] V. Puttasubbappa and H. Perros, "Performance analysis of limited-range wavelength conversion in an OBS switch", Telecommunications Systems Journal, vol. 31, no. 2-3, pp. 227-246, 2006.

[10] V. Eramo, M. Listanti, and M. Spaziani, "Resources sharing in optical packet switches with limited-range wavelength converters", J. Lightw. Technol., vol. 23, no. 2, pp. 671-687, 2005.

[11] M. Savi, C. Raffaelli, N. Akar, and E. Karasan, "Traffic analysis of synchronous buffer-less optical switch with shared limited range wavelength converters", in IEEE High Performance Switching and Routing (HPSR), New York, 2007.

[12] L. Kleinrock, Queuing Systems, Vol. 1, Theory, John Wiley, New York, 1975.

[13] G. H. Golub and C. F. van Loan, Matrix Computations, The Johns Hopkins University Press, 3rd edition, 1996.

[14] N. Akar and Y. Gunalay, "Stochastic analysis of finite population bufferless multiplexing in optical packet/burst switching systems", IEICE Trans. Commun., vol. E90-B(2), pp. 342-345, 2007. 